\title{
MAGNETIC DEVICE SIMULATION MODELLING AND OPTIMISATION FOR SCALE CONTROL
}

\author{
Lipus, L. C. ; Acko, B. ${ }^{*} \&$ Hamler, A.** \\ University of Maribor, Smetanova 17, SI-2000 Maribor, Slovenia \\ *Faculty of Mechanical Engineering \\ ${ }^{* *}$ Faculty of Electrical Engineering and Computer Science \\ E-Mail:lucija.crepinsek-lipus@uni-mb.si
}

\begin{abstract}
The paper presents model devices for magnetic water treatment, optimized for scale control at water capacities up to a few $\mathrm{m}^{3} / \mathrm{h}$. Different arrangements of permanent magnets were investigated and simulated at different water-flow capacities to yield the proper magnetic field distribution and the required magnetic flux density, using the computational program OPERA 15R1 (Vector Fields Software) with the finite-element method, considering the real 3Dgeometry, non-linearity of magnetic properties of construction materials, and the interaction of neighbouring poles.
\end{abstract}

(Received in October 2011, accepted in April 2012. This paper was with the authors 2 months for 1 revision.)

Key Words: Magnetic Water Treatment, Magnetic-Field Simulation, Three-Dimensional Model

\section{INTRODUCTION}

In water processing, the use of water is as important as the use of chemicals. Water acts as a heating medium, a transport medium, a solvent or as a wetting agent. It is generally ideal mineral solvent resulting in underground supplies reach with minerals from natural and even pollution origin [1-2]. Therefore, fouling is a frequent technological problem that requires proper scale-prevention treatment. The environmental and economic concerns that arise with traditional chemical treatments are strong motivation for the development of safer and cleaner physical methods, such as the application of permanent magnets [3-6], electromagnetic coils [7-10], electrodes [11], ultrasound [12] and catalytic metals [13]. Several operational parameters, e.g. water output temperature, water-flow velocity and magnetic/electric field parameters, must be considered in order to construct a proper device. Here a device with permanent magnets is modelled to find a technologically efficient and economically preferable solution, conceptionally considered as a product and a process technical innovation [14]. For this purpose, a review is given of the relevant parameters from several studies with static magnetic fields. According to these information and field experiences with selfconstructed magnetic devices for low water-flow capacities, an annular arrangement of permanent magnets on the outer side of the installation pipe is chosen and analyzed, to find an appropriate solution for higher water-flow capacities.

\section{EMPIRICAL DATA FOR DEVICE MODELLING}

Since the mineral salts are naturally present in water supplies (in fine suspended and dissolved form) the sparingly soluble salts, commonly calcium carbonate, often form a hard scale on the walls of pipe installations, requiring more pumping power because of a reduction in the flow area. Furthermore, in cooling-water applications, the fouling occurs abundantly on heated 
surfaces, reducing the heat-exchange efficiency because of the insulating properties of the scale. Thus, various scale-prevention and cleaning methods are used for fouling mitigation to help lessen, control, or prevent scaling. Traditional chemical methods, such as the preprecipitation of scale former with lime or soda ash, the addition of scale-inhibiting reagents and the replacement of the scale former with soluble ions by ion exchange, are effective but substantially change the solution chemistry. Although chemical additives have a high success rate, there are many disadvantages and concerns associated with their use. More stringent environmental laws have increased the costs associated with storage, handling and disposal. Accumulated chemical residues over a long period of time pose a concern for the environment. The common working principle of physical scale-control treatments is the preprecipitation of calcium carbonate (homogenous nucleation/coagulation in bulk water under the influence of the magnetic/electric field or ultrasound) into fine suspended particles that later (in bulk water under the hot conditions of the heat exchanger) offer preferable surfaces for crystallization and are deposited as a loosely adhered sludge, or are carried away from the heating system by the water flow.

The anti-scale magnetic treatment (AMT) of hard water has been employed for more than half a century, but the application has sometimes proven to be ineffective. Namely, in addition to the complexity of $\mathrm{CaCO}_{3}$ crystallization and its dependence on numerous treatment parameters, the working principle of AMT is far from magnetic-force action. In the physicochemical mechanism of water dispersion/solution, various influences may alter micellization/crystallization during exposure to the magnetic field, such as induced changes in hydration of ions and crystal surfaces, or magnetically induced proton transfer from hydrogen carbonate to water (owing to proton spin inversion in the field of diamagnetic salts) [15]. Additionally, Lorentz-force action on electrically charged particles, ions, molecules and surfaces occurs when water is conducted through the magnetic field [16]. Much experimental research under well-controlled laboratory conditions $[3,4,17,18]$ and field tests in real longterm conditions [19] were needed to determine the operational requirements that are essential for efficient scale control (Table I). An alternating magnetic field, orthogonal to the waterflow direction is more efficient. When a magnetic device is constructed and applied according to these requirements, small amounts of slimy, easily washable deposits are formed instead of hard carbonate scale and even old hard scale can gradually be removed after the AMT installation. Even at very hot and alkaline conditions, the precipitate on the heating surface can become thinner, less compact and easier to remove $[6,20]$.

Table I: Workload for AMT device modelling.

\begin{tabular}{|c|c|}
\hline Minimal magnetic flux density & $B=0.1$ to $0.2 \mathrm{~T}$ \\
\hline Recommended water flow velocity & $v=1$ to $2 \mathrm{~m} / \mathrm{s}$ \\
\hline Minimal exposure time & $\tau=0.1$ to $0.2 \mathrm{~s}$ \\
\hline
\end{tabular}

\section{OPTIMISATION WITH FINITE-ELEMENT SIMULATION}

The ability of any model to identify optimal configuration parameters depends on the accuracy of the model and on the representativeness of the workload. The workload definition is complex, and even harder to describe properly than the system itself because it should exactly describe the process of request arrival at the system components, for a given simulation [21].

Different arrangements of permanent magnets were investigated and simulated to find an economic solution for a particular water-flow capacity. After the preliminary dimensioning, the precise dimensions were sought to find the proper magnetic field distribution and the 
required magnetic flux density. The computational program OPERA 15R1 (Vector Fields Software), with the finite-element method (FEM) was used for this numerical simulation (other methods are possible too [22-23]).

Applied method enables precise consideration of real 3D-geometry, non-linearity of magnetic properties of construction materials, and interaction of neighbouring poles [24]. The calculations were made for a static magnetic field, based on Poisson's equation (1) in the region of permanent magnets, and on Laplace's equation (2) in the region of the all other parts of the magnetic device:

$$
\begin{gathered}
\nabla \times\left(\frac{1}{\mu} \nabla \times \mathbf{A}\right)=\frac{1}{\mu_{0}} \nabla \times \mathbf{B}_{\mathrm{r}} \\
\nabla \times\left(\frac{1}{\mu} \nabla \times \mathbf{A}\right)=0
\end{gathered}
$$

Here $\mathbf{A}$ is magnetic vector potential, $\mathbf{B}_{\mathrm{r}}$ is remaining magnetic flux density of permanent magnets, $\mu_{0}$ is the absolute permeability of material and $\mu$ is the permeability of vacuum $\left(4 \pi \cdot 10^{-7} \mathrm{Vs} / \mathrm{Am}\right)$. Because the magnetic field is conservative; the magnetic vector potential is defined by:

$$
\begin{gathered}
\mathbf{B}=\nabla \times \mathbf{A} \\
\nabla \cdot \mathbf{A}=0
\end{gathered}
$$

Here $\mathbf{B}$ is magnetic flux density.

Since the conditions in the cases of geometrically complex models cannot be determined directly by this equation, the geometry must be described by several divided simple geometry elements (finite elements). Considering the interference from neighbouring elements, a system of equations is derived, of which the solution is the value of $\mathbf{A}$. Because the potential $\mathbf{A}$ is defined as (3), the values and the distribution of magnetic flux density $\mathbf{B}$ can be found in every point of the FEM model by the post-processing of $\mathbf{A}$. The evaluation of $\mathbf{B}$ along a chosen line or plane yields a local distribution of B. On the basis of these local distributions, proper configuration for device construction can be selected.

Additionally, the local distributions are the criteria for the magnetic system optimisation. In our case, the optimisation was performed by a stochastic procedure of seeking the construction that is the most convenient. The following parameters were varied: the thickness of permanent magnets (i.e., the dimension orthogonal to the water-flow direction); the width (i.e. the dimension parallel to the water-flow direction); the direction of the magnets' magnetisation; the pipe diameter (which influences the distance between the magnets); the distances among the magnetic poles and the thickness of the magnetic yoke. The aim of the procedure was to provide the peak magnetic flux density in the water that is required for efficient AMT (taken from Table I), and the homogeneity of the field along the cross-section of the pipe in the middle of the magnetic unit. In all cases, NdFeB magnets with a remaining magnetic flux density of $B_{\mathrm{r}}=1.12 \mathrm{~T}$ and a coercive magnetic field intensity of $H_{\mathrm{c}}=781 \mathrm{kA} / \mathrm{m}$ were used.

For the material of the magnetic yoke, low-carbonic steel was selected; and the pipe was from non-magnetic material. The external pipe diameter was according to standards; the water-flow capacity was calculated at a wall thickness of approximately $2 \mathrm{~mm}$, but the thickness is subject of to the pressure load in the pipeline and may be slightly changed, since the field distribution remains the same, due to the non-magnetic properties of the wall. 


\section{RESULTS AND DISCUSSION}

Permanent magnets are sufficiently strong and thermally stable to be used for the construction of devices providing a magnetic field as prescribed for efficient AMT. The alternating arrangement of rectangular magnets along the pipe produces magnetic lines transversal to the water flow. For small water-flow capacities, such as in the majority of laboratory tests and domestic sanitation pipelines, a rectangular gap is applicable. The dimensions of the rectangular gap are adjusted to the pipe diameter and determined by the numerical simulation for the required minimum magnetic-flux density, $0.2 \mathrm{~T}$, and the limit for water velocity (see Table I). By increasing $B$, the cross-section available for water conduction is reduced. The length of the magnetic gap is calculated simply from the water velocity for the required minimum exposure of water to the magnetic field; then the number of successive units is determined for the given length of one magnet.

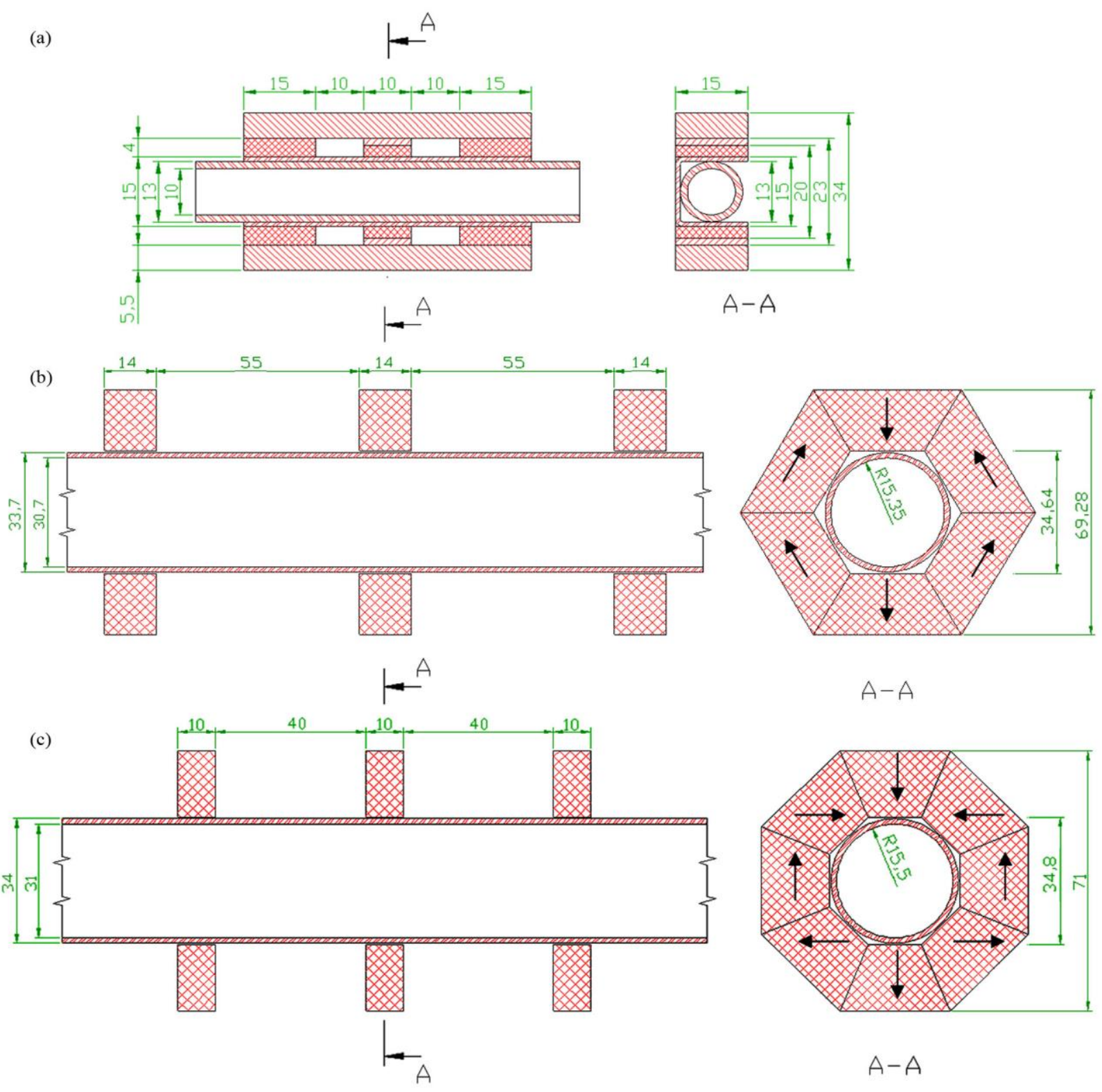

Figure 1: Permanent magnets on water pipe: (a) two rows of square magnets,

(b) hexagonal configuration of magnets, (c) octagonal configuration of magnets. 
In the cases of low-diameter water pipes with a common water velocity from 1 to $1.5 \mathrm{~m} / \mathrm{s}$, two rows of alternating magnets can be simply put onto opposite sides of the pipe (Fig. 1a). At an inner pipeline diameter of $10 \mathrm{~mm}$, the flow capacity is 0.3 to $0.4 \mathrm{~m}^{3} / \mathrm{h}$.

In the cases from 0.5 to $2 \mathrm{~m}^{3} / \mathrm{h}$, a magnetic device with a rectangular gap (as wide as the inner diameter of the pipeline) is convenient. The gap height is subject to numerical simulation for the required magnetic flux density (from Table I). When water moves from the spherical into the smaller area of the rectangular cross-section, the velocity rises; thus the upper value $2 \mathrm{~m} / \mathrm{s}$ (from Table I) limits the application for higher flow capacities.

Furthermore, at capacities of a few $\mathrm{m}^{3} / \mathrm{h}$, with common water velocity from 1.5 to $2 \mathrm{~m} / \mathrm{s}$, hydrodynamic resistance has to be taken into consideration. For this reason, another configuration of permanent magnets was tried and several modelling solutions were found. Two optimized cases are presented here: a hexagonal configuration (Fig. 1b) and an octagonal configuration (Fig. 1c), for the range from 4 to $5.5 \mathrm{~m}^{3} / \mathrm{h}$.
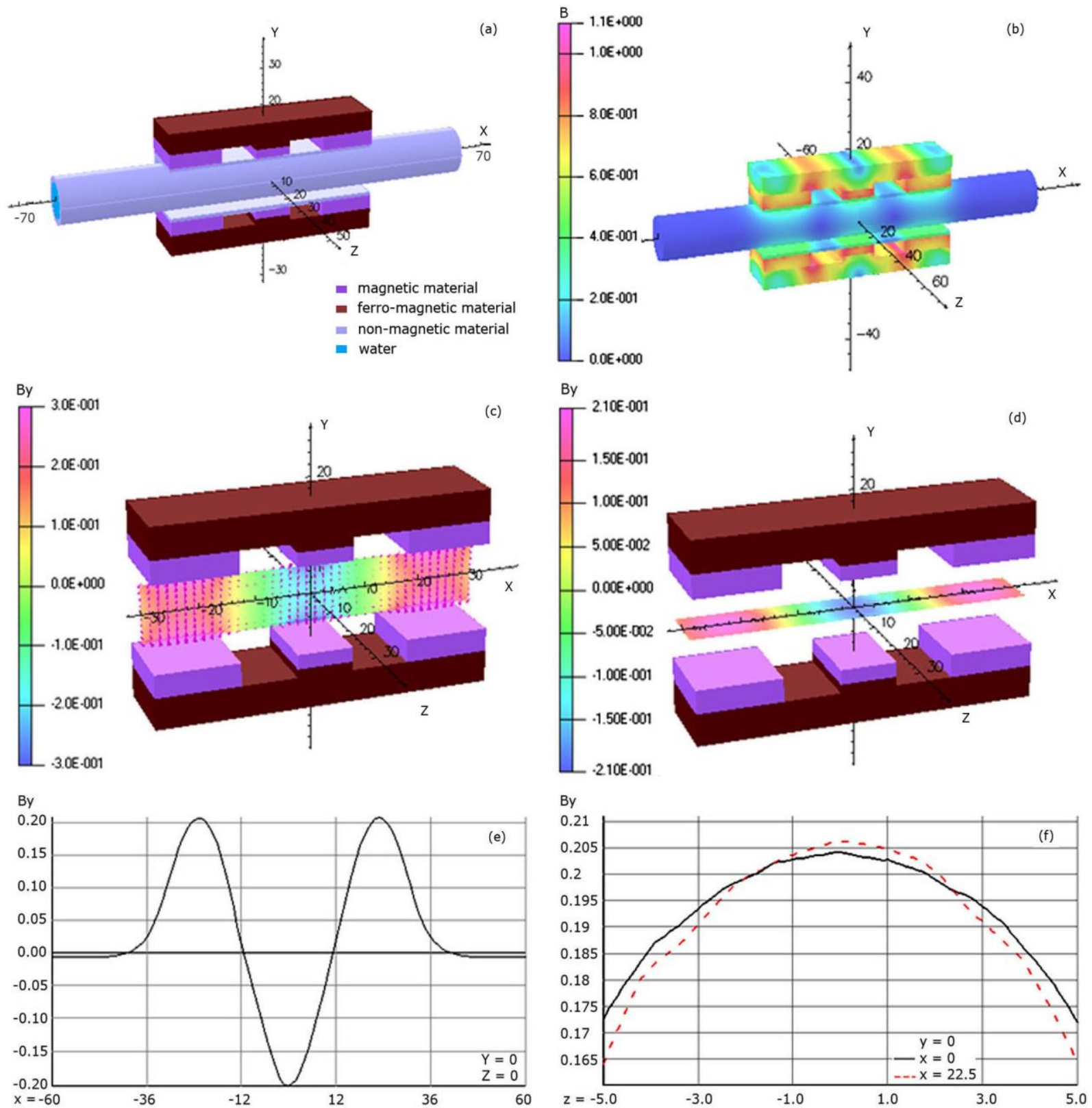

Figure 2: Magnetic flux distribution for the set of two parallel rows of square magnets. 
For extremely high water-flow capacities, an annular gap and electromagnetic coils are preferable [10].

Fig. 2 presents the magnetic flux distribution for the set of two parallel rows of square magnets (dimensions from Fig. 1a). The magnets are transversally magnetized and adjusted on nonmagnetic pipe with U-yoke (Fig. 2a). The ferromagnetic plate-yokes concentrate the magnetic flux (Fig. 2b) and consequently intensify the magnetic flux density in the water region, where they are orientated orthogonally to the water flow (in the Y-direction, Fig. 2c). The peak of magnetic flux density in the water occurs at the centre of each magnetic pair and alternates, owing the alternating orientation of magnets along the pipe (Fig. 2e). The field along the cross-section of the pipe is semi-homogeneous (Fig. $2 \mathrm{~d}, \mathrm{f}$ ).
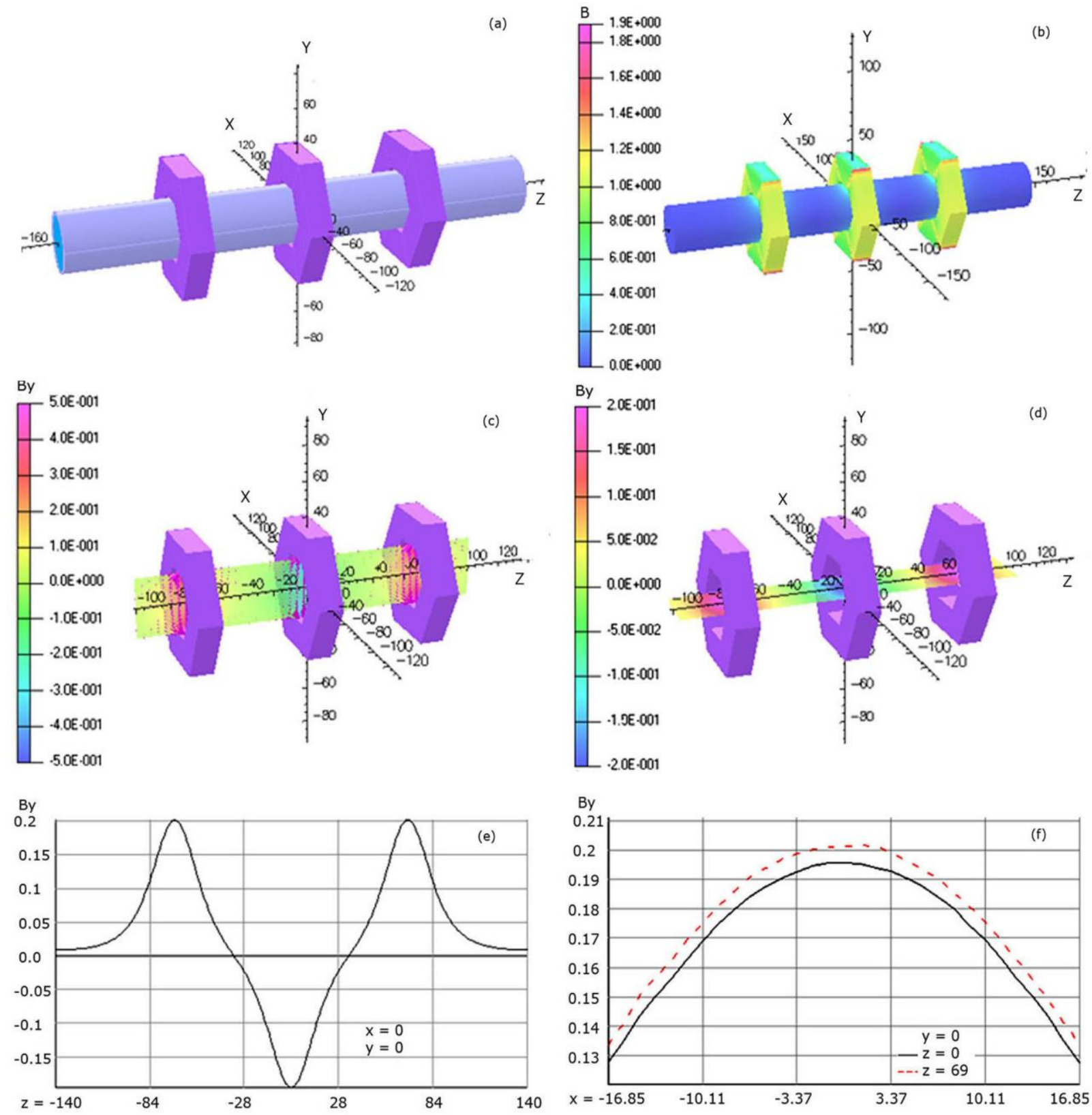

Figure 3: Magnetic flux distribution for the set of hexagonal-configured magnets.

Fig. 3 presents the magnetic flux distribution for the set of magnets with hexagonal configuration that are magnetized as presented in Fig. 1b. Although the pipe has a diameter 3 times higher, the field yielded is similar to the former case. The interaction among 
neighbouring hexagonal units is mitigated by thickening the magnets and raising the distance between the units. The octagonal configuration (with dimensions and magnetization from Fig. 1c) yields a similar magnetic field distribution (Fig. 4), but somewhat stronger near the peak (Fig. 3f).
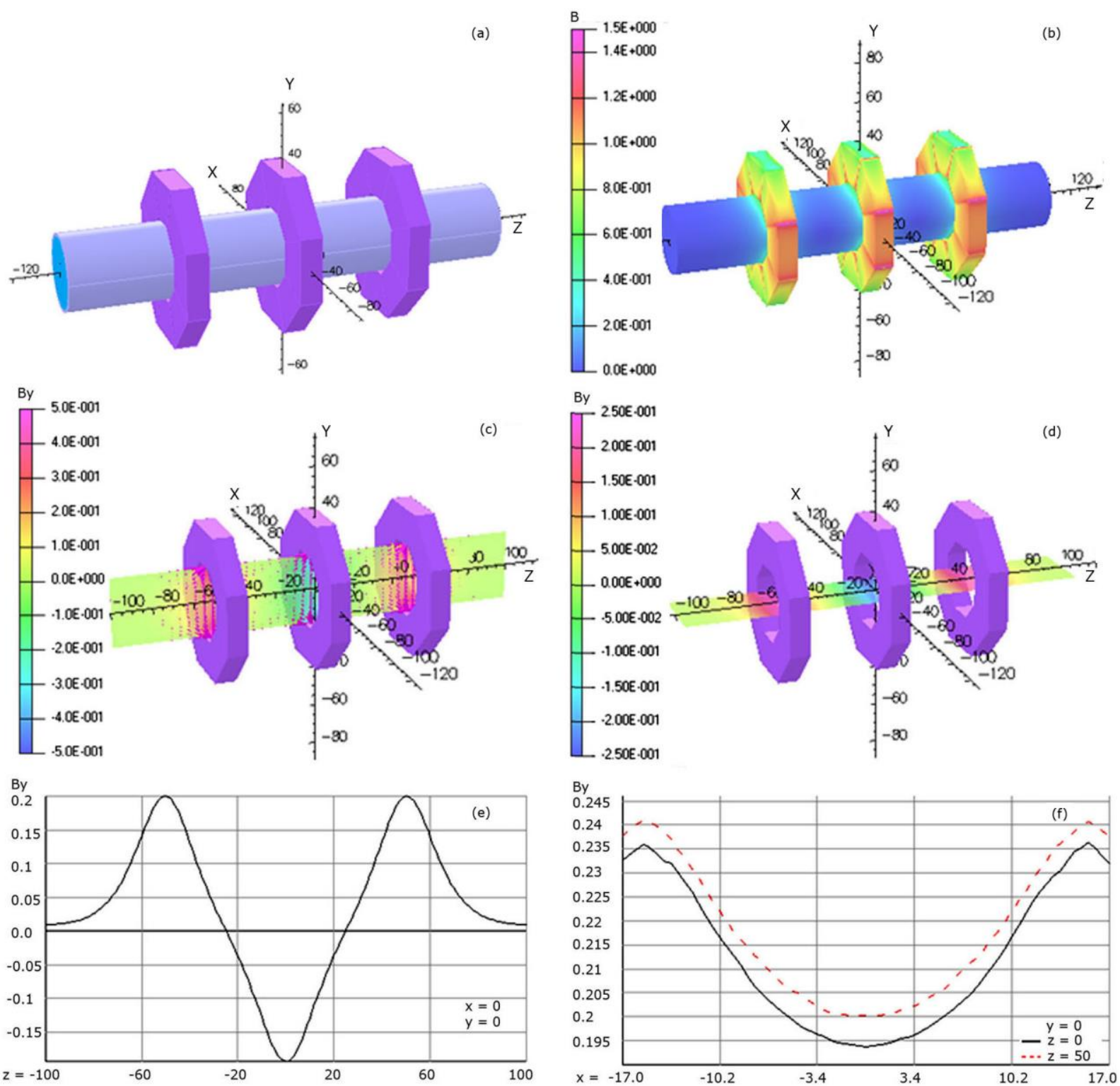

Figure 4: Magnetic flux distribution for the set of octagonal-configured magnets.

\section{CONCLUSION}

This paper gives a preview of the development of magnetic devices for scale control. Modelling and simulation are based on a workload of operational requirements for the technological process and of the material characteristics for the device construction. Vector Fields Software with the finite-element method enabled precise observation of the magnetic field. Among several results for different water-flow capacities, models of magnetic array on non-magnetic pipe were presented: parallel rows of square magnets convenient for small capacities less than $0.5 \mathrm{~m}^{3} / \mathrm{h}$; octagonal and hexagonal arrays for higher capacities, from 4 to $5.5 \mathrm{~m}^{3} / \mathrm{h}$. For intermediate capacities, the model with a rectangular gap yields the required 
magnetic field, but produces some hydrodynamic pressure loss. However, application of such devices instead of chemical scale-prevention treatment proved ecologically and economically advantageous in low-capacity water processing. The construction and installation is relatively easy and also applicable for removal of previously formed scale, applicable also for muddy waters, while the life-time is long and without any energy consumption.

\section{ACKNOWLEDGEMENT}

The model of hexagonal/octagonal-configured magnets was developed in the frame of Eureka project E! 4956 Magnet, co-financed by the Ministry of Economic Development and Technology, Slovenia.

\section{REFERENCES}

[1] Stumm, W.; Morgan, J. J. (1996). Aquatic Chemistry: Chemical Equilibria and Rates in Natural Waters. $3^{\text {th }}$ ed., Wiley-Interscience Publication, New York

[2] Sperac, M.; Dimter, S. (2010). Transport of pollutants in seepage water from pavement structures with inbuilt fly ash, Technical Gazette, Vol. 17, No. 2, 231-236

[3] Alimi, F.; Tlili, M. M.; Ben Amor, M.; Maurin, G.; Gabrielli, C. (2009). Effect of magnetic water treatment on calcium carbonate precipitation: Influence of the pipe material, Chemical Engineering and Processing, Vol. 48, No. 8, 1327-1332, doi:10.1016/j.cep.2009.06.008

[4] Chang, M. C.; Tai, C. Y. (2010). Effect of the magnetic field on the growth rate of aragonite and the precipitation of $\mathrm{CaCO}_{3}$, Chemical Engineering Journal, Vol. 164, No. 1, 1-9, doi:10.1016/j.cej.2010.07.018

[5] Stuyven, B.; Vanbutsele, G.; Nuyens, J.; Vermant, J.; Martens, J. A. (2009). Natural suspended particle fragmentation in magnetic scale prevention device, Chemical Engineering Science, Vol. 64, No. 8, 1904-1906, doi:10.1016/j.ces.2008.12.022

[6] Lipus, L. C.; Dobersek, D. (2007). Influence of magnetic field on the aragonite precipitation, Chemical Engineering Science, Vol. 62, No. 7, 2089-2095, doi:10.1016/j.ces.2006.12.051

[7] Lee, S. H.; Cho, Y. I. (2002). Velocity effect on electronic-antifouling technology to mitigate mineral fouling in enhanced-tube heat exchanger, International Journal of Heat and Mass Transfer, Vol. 45, No. 20, 4163-4174, doi:10.1016/S0017-310(02)00104-7

[8] Xing, X. K. (2008). Research on the electromagnetic anti-fouling technology for heat transfer enhancement, Applied Thermal Engineering, Vol. 28, No. 8-9, 889-894, doi:10.1016/ j.applthermaleng.2007.07.006

[9] Shahryari, A.; Pakshir, M. (2008). Influence of a modulated electromagnetic field on fouling in a double-pipe heat exchanger, Journal of Materials Processing Technology, Vol. 203, No. 1-3, 389-395, doi:10.1016/j.jmatprotec.2007.10.048

[10] Lipus, L. C.; Acko, B.; Hamler, A. (2011). Electromagnets for high-flow water processing, Chemical Engineering and Processing, Vol. 50, No. 9, 952-958, doi:10.1016/j.cep.2011.07.004

[11] Tijing, L. D.; Kim, H. Y.; Lee, D. H.; Kim, C.S.; Cho, Y. I. (2010). Physical water treatment using RF electric fields for the mitigation of $\mathrm{CaCO}_{3}$ fouling in cooling water, International Journal of Heat and Mass Transfer, Vol. 53, No. 7-8. 1426-1437, doi:10.1016/ j.ijheatmasstransfer.2009.12.009

[12] Al Nasser, W. N.; Pitt, K.; Hounslow, M. J.; Salman, A. D. (2012). Monitoring of aggregation and scaling of calcium carbonate in the presence of ultrasound irradiation using focused beam reflectance measurement, Powder Technology, In Press, doi:10.1016/j.powtec.2012.03.021

[13] Tijing, L. D.; Yu, M.-H.; Kim, C.-H.; Amarjargal, A.; Lee, Y. C.; Lee, D. H.; Kim, D. W.; Kim, C. S. (2011). Mitigation of scaling in heat exchangers by physical water treatment using zinc and tourmaline, Applied Thermal Engineering, Vol. 31, No. 11-12, 2025-2031, doi:10.1016/ j.applthermaleng.2011.03.011 
[14] Palcic, I.; Buchmeister, B.; Polajnar, A. (2010). Analysis of Innovation Concepts in Slovenian Manufacturing Companies, Strojniški vestnik - Journal of Mechanical Engineering, Vol. 56, No. $12,803-810$

[15] Madsen, H. E. L. (2007). Theory of electrolyte crystallization in magnetic field, Journal of Crystal Growth, Vol. 305, No. 1, 271-277, doi:10.1016/j.jcrysgro.2007.04.023

[16] Lipus, L. C.; Krope, J.; Crepinsek, L. (2001). Dispersion destabilization in magnetic water treatment, Journal of Colloid and Interface Science, Vol. 236, No. 1, 60-66, doi:10.1006/ jcis. 2000.7392

[17] Gabrielli, C.; Jaouhari, R.; Maurin, G.; Keddam, M. (2001), Magnetic water treatment for scale prevention, Water Research, Vol. 35, No. 13, 3249-3259, doi:10.1016/S0043-1354(01)00010-0

[18] Coey, J. M. D.; Cass, S. (2000). Magnetic water treatment, Journal of Magnetism and Magnetic Materials, Vol. 209, No. 1-3, 71-74, doi:10.1016/S0304-8853(99)00648-4

[19] Szkatula, A.; Balanda, M.; Kopec, M. (2002). Magnetic treatment of industrial water. Silica activation, European Physical Journal - Applied Physics, Vol. 18, No. 1, 41-49, doi:10.1051/ epjap:2002025

[20] Kozic, V.; Hamler, A.; Ban, I.; Lipus, L. C. (2010). Magnetic water treatment for scale control in heating and alkaline conditions, Desalination and Water Treatment, Vol. 22, No. 1-3, 65-71, doi:10.5004/dwt.2010.1549

[21] Vickovic, L.; Celar, S.; Mudnic, E. (2011). Disk array simulation model development, International Journal of Simulation Modelling, Vol. 10, No. 1, 27-37, doi:10.2507/IJSIMM10(1)3.174

[22] Gusel, L.; Rudolf, R.; Kosec, B. (2009). Analysis of a strain rate field in cold formed material using the visioplasticity method, Metalurgija - Metallurgy, Vol. 48, No. 2, 103-107

[23] Obrovic, B. R.; Savic, S. R.; Petrovic, R. S. (2010). Ionized gas boundary layer on bodies of revolution in the presence of magnetic field, Technical Gazette, Vol. 17, No. 1, 35-42

[24] Jesenik, M., Gorican, V., Trlep, M., Hamler, A.; Stumberger, B. (2006). Finite element magnetic field calculation in the anisotropic magnetic material, Przeglęad Elektrotechniczny, Vol. 82, No. $5,20-22$ 